\title{
Phase mixing of a three dimensional magnetohydrodynamic pulse
}

\author{
D. Tsiklauri, V. M. Nakariakov, and G. Rowlands
}

Physics Department, University of Warwick, Coventry, CV4 7AL, UK

Received 4 October 2002 / Accepted 16 December 2002

\begin{abstract}
Phase mixing of a three dimensional magnetohydrodynamic (MHD) pulse is studied in the compressive, threedimensional (without an ignorable coordinate) regime. It is shown that the efficiency of decay of an Alfvénic part of a compressible MHD pulse is related linearly to the degree of localization of the pulse in the homogeneous transverse direction. In the developed stage of phase mixing (for large times), coupling to its compressive part does not alter the power-law decay of an Alfvénic part of a compressible MHD pulse. The same applies to the dependence upon the resistivity of the Alfvénic part of the pulse. All this implies that the dynamics of Alfvén waves can still be qualitatively understood in terms of the previous $2.5 \mathrm{D}$ models. Thus, the phase mixing remains a relevant paradigm for the coronal heating applications in the realistic $3 D$ geometry and compressive plasma.
\end{abstract}

Key words. magnetohydrodynamics (MHD) - waves - Sun: activity - Sun: Corona

\section{Introduction}

The phase mixing of incompressible Alfvén waves (Heyvaerts \& Priest 1983) has been intensively studied as a possible mechanism of coronal heating in open magnetic structures of the polar regions of the Sun. Also, there are recent observational indications that the same mechanism operates in coronal loops Ofman \& Aschwanden (2002). In a compressible plasma, phase mixing of linearly polarized plane Alfvén waves may provide enhanced heating through the nonlinear generation of fast magnetoacoustic waves. This phenomenon has been intensively studied by Malara et al. (1996), Nakariakov et al. (1997, 1998), Botha et al. (2000), Tsiklauri et al. $(2001,2002)$ both analytically and numerically.

There are three main motivations for further development of this work: the first, as was recently shown by Hood et al. (2002a), is that phase mixing of localized single Alfvén pulses results in a slower, power-law damping as opposed to the standard $\propto \exp \left(-t^{3}\right)$ one for harmonic Alfvén waves (Heyvaerts \& Priest 1983). This suggests that localized Alfvénic perturbations can transport energy higher into the corona than harmonic ones. Also, Hood et al. (2002b) showed that this powerlaw damping is replaced by even faster power-law decay as the number of pulses is increased. Such perturbations could be generated e.g. by transient events such as solar flares, coronal mass ejections, etc. (Roussev et al. 2001). The second follows from the recent demonstration by Tsiklauri \& Nakariakov (2002) that in the linear regime and in 3D geometry,

Send offprint requests to: $\mathrm{D}$. Tsiklauri,

e-mail: tsikd@astro.warwick.ac.uk generation of compressive perturbations significantly modifies the dynamics of the Alfvénic part of a MHD pulse. In fact, while solving the propagation part of the problem, i.e. considering an ideal plasma limit, it has been shown that an initially localized 3D Alfvén pulse interacting with a 1D transverse inhomogeneity evolves to a MHD pulse with a significant compressible component. This 3D MHD pulse is subject to phase mixing. Therefore, as a natural step forward, in this study we include a finite plasma resistivity in order to investigate quantitatively dissipation of a 3D MHD pulse via phase mixing. The third follows from Parker's (1991) earlier suggestion that in order for the phase mixing mechanism to work, it requires the presence of a third ignorable coordinate. Although it has been justly observed that having an ignorable coordinate seems unlikely in a filamentary corona, and, also, in the absence of an ignorable coordinate, the "Alfvén-type waves" are coupled to the "fast magnetosonic-type modes", we believe it was not justified to dismiss the importance of phase mixing without a rigorous, fully three dimensional study.

\section{The model}

In our model we use the MHD equations

$\rho \partial_{t} \boldsymbol{V}+\rho(\boldsymbol{V} \cdot \nabla) \boldsymbol{V}=-\nabla p-(4 \pi)^{-1} \boldsymbol{B} \times \operatorname{curl} \boldsymbol{B}$,

$\partial_{t} \boldsymbol{B}=\operatorname{curl}(\boldsymbol{V} \times \boldsymbol{B})+\eta \Delta \boldsymbol{B}$,

$\partial_{t} p+\boldsymbol{V} \cdot \nabla p+\gamma p \nabla \cdot \boldsymbol{V}=(\gamma-1) \eta(\operatorname{curl} \boldsymbol{B})^{2}$,

where $\boldsymbol{B}$ is the magnetic field, $\boldsymbol{V}$ is the plasma velocity, $\rho$ is the plasma mass density, and $p$ is the plasma thermal pressure. 


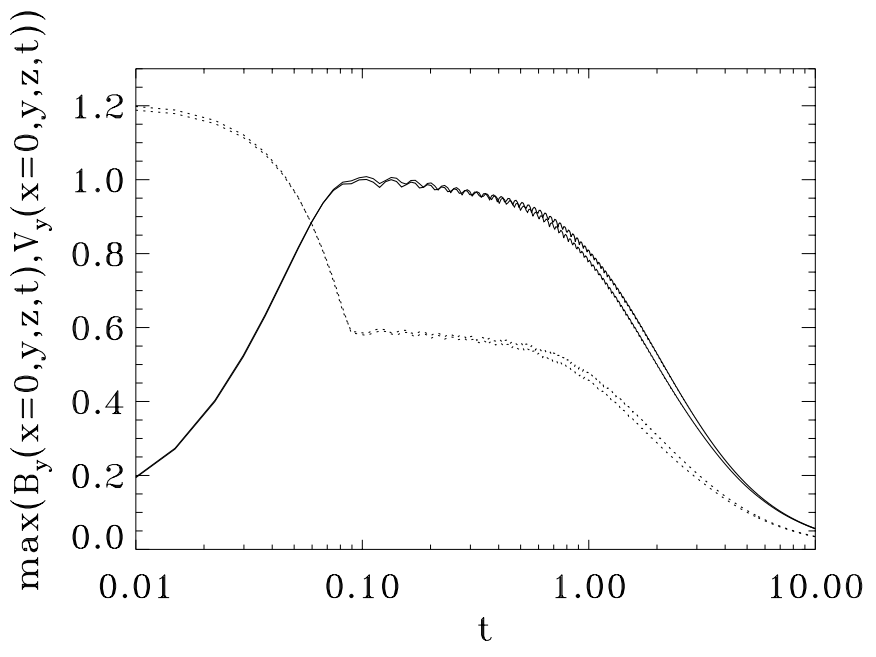

Fig. 1. Time evolution of $\max \left(B_{y}(x=0, y, z, t)\right.$ (solid lines) and $\max \left(V_{y}(x=0, y, z, t)\right.$ (dotted lines). Note the plot is log-normal. Here, $\alpha_{y}=1.0$. The thick lines represent simulation results with the doubled resolution in $y$-direction. Note progressive convergence of the solutions with the increase of simulation time.

In what follows we use 5/3 for the value of $\gamma$. Also, here $\eta$ denotes plasma resistivity.

We solve Eqs. (1)-(3) in Cartesian coordinates $(x, y, z)$. Note that we solve a fully $3 \mathrm{D}$ problem retaining variation in the $y$-direction, i.e. $(\partial / \partial y \neq 0)$. A uniform magnetic field $B_{0}$ is in the $z$-direction. The plasma configuration has a onedimensional inhomogeneity in the equilibrium density $\rho_{0}(x)$ and temperature $T_{0}(x)$, while the unperturbed thermal pressure, $p_{0}$, is taken to be constant everywhere.

\section{Numerical results}

Evolution of a purely Alfvénic perturbation allows a full analytical treatment and this is presented in Appendix A. However, if an initial Alfvénic perturbation depends on the $y$-coordinate, contrary to the case studied in the Appendix A, the linearized version of the system of Eqs. (1)-(3) no longer allows decoupling of Alfvénic and compressive perturbations. In fact, all three modes, Alfvén, fast and slow magnetosonic, are intercoupled. Thus, they cannot be separated. Because of the complexity of the problem in this case, we resort to direct numerical simulations of Eqs. (1)-(3) using the numerical code Lare3d (Arber et al. 2001). We employ the following initial conditions:

$\left.V_{y}\right|_{t=0}= \begin{cases}-A C_{\mathrm{A}}(x)[1+\cos (10 \pi z)] & \\ \times \exp \left[-\alpha_{y}^{2}\left(y+\frac{1}{\sqrt{2} \alpha_{y}}\right)^{2}\right] & |z| \leq 0.1, \\ 0 & \text { elsewhere. }\end{cases}$

The rest of the perturbations at $t=0$ are set to zero (including $B_{y}$, in this way we guarantee fulfillment of $\operatorname{div} \boldsymbol{B}=0$, at $t=0)$. In Eq. (4) $A$ is the amplitude of the wave, $C_{\mathrm{A}}(x)$ is the local Alfvén speed (see Appendix A) and $\alpha_{y}$ is a free parameter that controls the strength of the gradient in the $y$ direction of the initial perturbation. In effect, $\alpha_{y}$ controls the strength of the coupling between the Alfvénic and compressive (fast and slow magnetosonic) components of the MHD pulse. This particular

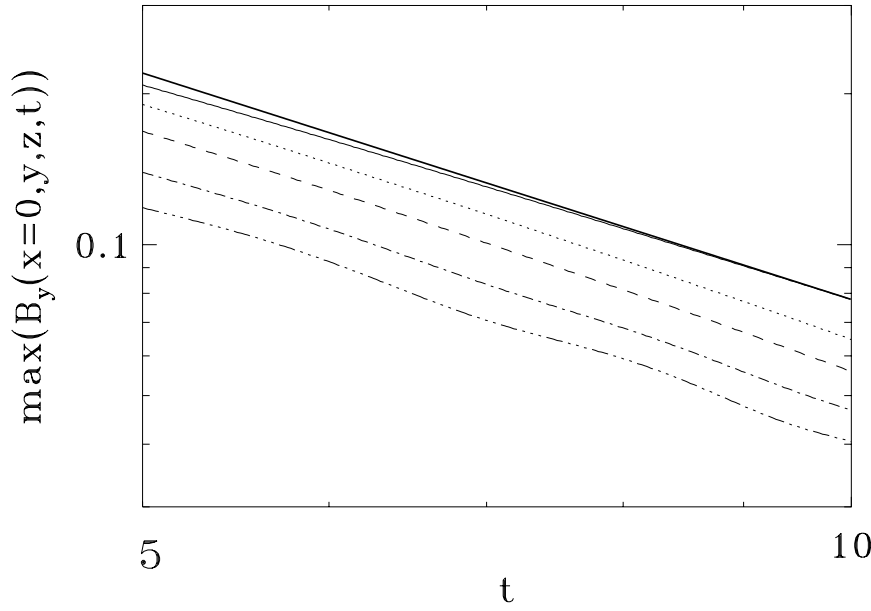

Fig. 2. The developed stage of phase mixing: time evolution of $\max \left(B_{y}(x=0, y, z, t)\right.$ for large times (log-log plot). The thick solid line represents the analytic solution (Eq. (A.10) with an additional pre-factor 0.5 , to account for the split of the initial pulse into two D'Alambert solutions), while the thin line is our numerical simulation result (for $\alpha_{y}=0$ ). Note that the deviation between the two progressively vanishes. The dotted, dashed, dash-dotted and dashtriple-dotted curves correspond to the numerical solutions for $\alpha_{y}=$ $0.5,1.0,1.5,2.0$ respectively.

choice of $y$-dependence in Eq. (4) is dictated by a desire to have the maximum of the absolute value of $\partial_{y} V_{y}$, which is responsible for the coupling to the compressive modes, at the middle of the simulation cube, where the background plasma inhomogeneity is the strongest. Since Lare $3 d$ is a fully non-linear numerical code, we simply set small (linear) initial amplitude of the perturbation (given by Eq. (4)) as $A=0.0001$, while the output results are normalized to $A$. The simulation cube size was set by the limits $-11.0 \leq x, y, z \leq 11.0$. Boundary conditions used in all our simulations are zero-gradient in all three spatial dimensions. In fact, our strategy was to forbid any generated wave fronts to reach the boundaries to avoid contamination of the solution. This of course severely restricts simulation end time. However, with the computational resources at our disposal, it was still possible to reach the developed stage of phase mixing. In all our numerical simulations we fix the plasma $\beta$ at $10^{-3}$ (which is a plausible value for solar corona) and dimensionless resistivity (see Appendix A), $\eta$, at $5 \times 10^{-4}$ (which is the same value used by Hood et al. 2002a) unless stated otherwise. In Fig. 1 we track the evolution of the maximal value (over the whole simulation cube at a given time) of $B_{y}$ and $V_{y}$ at the middle of the density inhomogeneity at $x=0$, where phase mixing significantly enhances the dissipation. Note that $\max \left(\left|V_{y}(x=0, y, z, t)\right|\right)$ at $t=0$ is not unity as it is normalized to $A$, not to $\max \left(\left|V_{y}(x=0, y, z, t=0)\right|\right)$. We gather from this graph that although $B_{y}$ was initially zero, it is rapidly generated (on the time scale of $\sim 0.05$, which is the ratio of length-scale of the pulse $1 / 10 \pi$ to $\left.C_{\mathrm{A}}(0)=0.6\right)$, and subsequently it decays due to phase mixing. Most of our numerical runs were done with $200 \times 72 \times 400$ resolution (with a non-uniform grid), and we checked convergence of our results by doubling the number of grid points in the $y$-direction. Coincidence of the two runs was satisfactory and, in fact, 


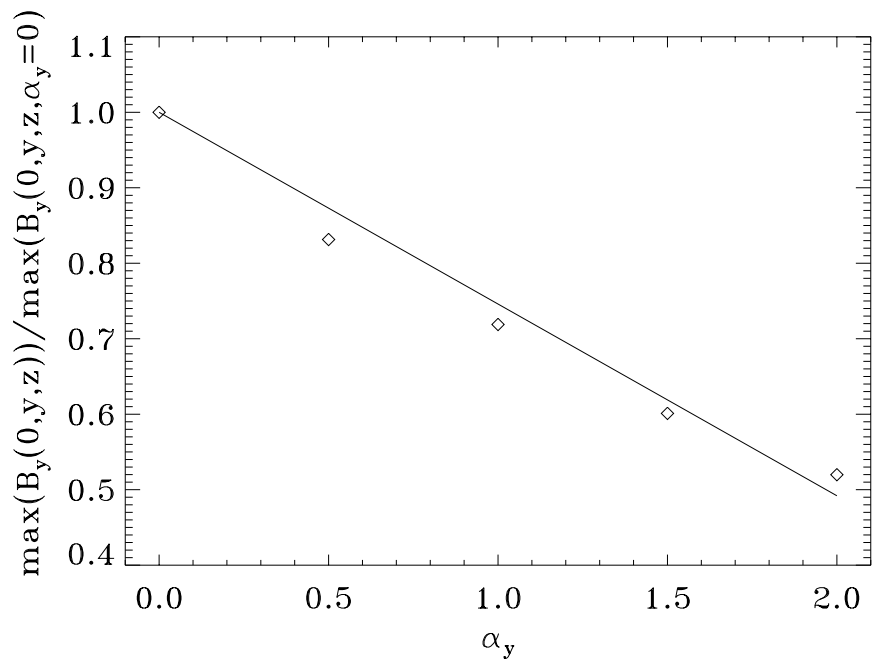

Fig.3. Efficiency of decay of the Alfvénic part of MHD pulse, $\max \left(B_{y}(x=0, y, z) / \max \left(B_{y}\left(x=0, y, z, \alpha_{y}=0\right)\right.\right.$, as a function of coupling to the compressive modes strength parameter, $\alpha_{y}$ at $t=10$.

it progressively improves with the increase of the simulation time. Although we were unable to double the resolution in $x$ and $z$, proof of the convergence of our results comes from the fact (see Fig. 2) that the analytic result (given by Eq. (A.10) with an additional pre-factor 0.5 to account for the splitting of initial $V_{y}$ perturbation into two D'Alambert solutions, Tsiklauri $\&$ Nakariakov 2002) and the numerical solution in the case $\alpha_{y}=0$ (when there is a complete decoupling of the Alfvén and the compressive modes) coincide perfectly for large $t$ s. In fact, at $t=9.998143$, the absolute deviation between the two is $(0.077836-0.077785) / 0.077785=0.0006$, which is a remarkably good match for a fully three dimensional simulation. Figure 2 demonstrates that: (A) an increase in the coupling strength parameter, $\alpha_{y}$, results in an overall decrease of the Alfvénic part $\left(B_{y}\right)$ of the MHD pulse, as expected, and (B) At large times, all lines on the log-log plot tend to become parallel. Therefore, the amplitude of the Alfvénic component of the MHD pulse decays with the same power-law $\left(B_{y} \propto t^{-3 / 2}\right)$ as in the case of a localized, pure, Alfvén wave (which occurs only when $\alpha_{y}=0$ ), i.e. coupling to the compressive waves does not affect the decay power-law of the Alfvénic part of the MHD pulse. In order to investigate and quantify point (A) further, in Fig. 3 we plot the efficiency of decay of the Alfvénic part of the MHD pulse, which we define as $\max \left(B_{y}(x=0, y, z) / \max \left(B_{y}\left(x=0, y, z, \alpha_{y}=0\right)\right.\right.$, as a function of coupling to the compressive modes strength parameter, $\alpha_{y}$ at $t=10$. Open diamonds represent numerical simulation results, while a straight line is the best fit, 1.0-0.25403 $\alpha_{y}$. We conclude that the efficiency of decay depends linearly upon the degree of localization of the pulse in the homogeneous transverse direction, i.e. the coupling strength parameter, $\alpha_{y}$. As far as point (B) is concerned, apart from the visual manifestation that at large times all lines tend to become parallel to each other, an actual fitting procedure also confirms that, indeed, amplitude of the Alfvénic part of the MHD pulse decays as a power-law $\left(\propto t^{-3 / 2}\right)$ within a few percent error to the powerlaw index. This is not a trivial result. From this we reach the

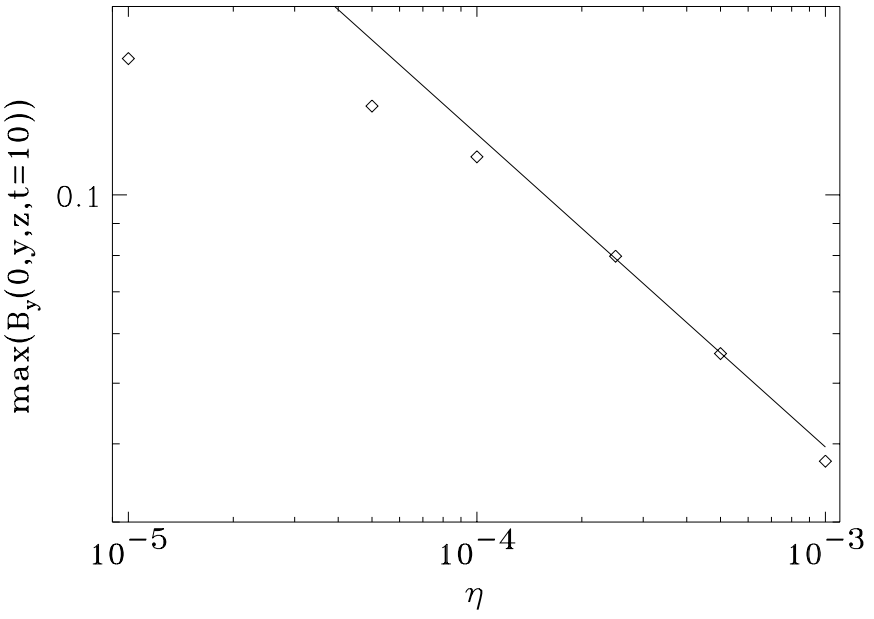

Fig. 4. Dependence of $\max \left(B_{y}(x=0, y, z, t=10)\right.$ upon resistivity $\eta$. The solid line represents the analytic solution (Eq. (A.10) with an additional pre-factor 0.5 , to account for the split of the initial pulse into two D'Alambert solutions and a further pre-factor 0.7188 , which comes from the middle point of Fig. 3), while open diamonds are numerical simulation results. Here, $\alpha_{y}=1.0$.

conclusion that the dynamics (phase mixing) of weakly nonplane Alfvén waves can still be qualitatively understood in terms of the previous $2.5 \mathrm{D}$ models. A certain level of understanding of this profound conclusion can be achieved by analyzing a linearized version of the system of Eqs. (1)-(3) for $\beta=0$. In this case, coupling between the Alfvén $\left(B_{y}\right)$ and fast magnetosonic mode $\left(B_{x}\right)$ (the slow mode is absent in this approximation) is described by the following system of equations:

$$
\left[\partial_{t t}^{2}-C_{\mathrm{A}}^{2}(x)\left(\partial_{x x}^{2}+\partial_{z z}^{2}\right)\right] B_{x}=\eta \partial_{t} \partial_{x x}^{2} B_{x}+C_{\mathrm{A}}^{2}(x) \partial_{x y}^{2} B_{y}
$$

$$
\left[\partial_{t t}^{2}-C_{\mathrm{A}}^{2}(x)\left(\partial_{y y}^{2}+\partial_{z z}^{2}\right)\right] B_{y}=\eta \partial_{t} \partial_{x x}^{2} B_{y}+C_{\mathrm{A}}^{2}(x) \partial_{x y}^{2} B_{x}
$$

From Eqs. (5) and (6) it follows that the terms responsible for the coupling between the Alfvén and fast magnetosonic mode are $C_{\mathrm{A}}^{2} B_{x, y} /\left(L_{x} L_{y}\right)$ (where $L_{x, y}$ are spatial scales in $x$ and $y$ ), while the terms responsible for the dissipation are $\eta B_{x, y} /\left(t_{\mathrm{A}} L_{x}^{2}\right)$ (where $t_{\mathrm{A}}$ is a typical Alfvén time scale). At large $t \mathrm{~s}$, because of the action of phase mixing in the region of density inhomogeneity, dissipative terms $\left(\propto L_{x}^{-2}\right)$ are much greater than the coupling terms $\left(\propto L_{x}^{-1}\right)$. Therefore, for $t \gg 1$ the dynamics of $B_{y}$ is governed by the dissipation rather than its coupling to the compressive fast mode. This, serves as an explanation why, at large times, all lines in Fig. 2 tend to become parallel, i.e. the amplitude of Alfvénic component of the MHD pulse decays as the same power-law $\left(B_{y} \propto t^{-3 / 2}\right)$ as in the case of localized, pure, Alfvén wave.

Also, we investigated the dependence of the Alfvénic part of the MHD pulse, which we quantify by $\max \left(B_{y}(x=0, y, z\right.$, $t=10$ ), upon resistivity, $\eta$. The results are presented in Fig. 4. On the one hand, an accurate analytic solution (A.10) for $B_{y}$, which scales as $\propto 1 / \sqrt{\eta}$, was obtained for the case of $\alpha_{y}=0$. On the other hand, point (B), mentioned above, indicates that when $\alpha_{y} \neq 0$, the solution (A.10) should be altered by a further pre-factor that can be determined e.g. from Fig. 3 (in this 


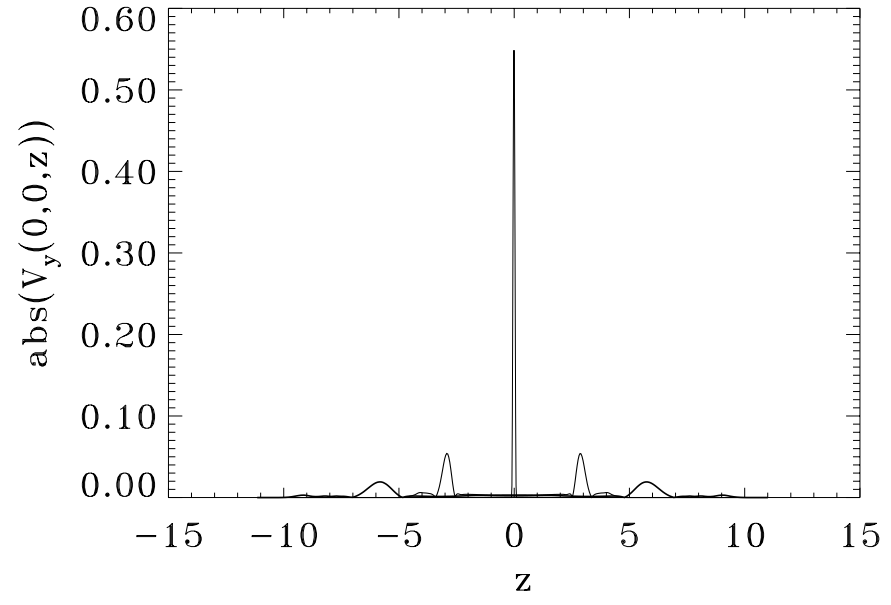

Fig. 5. Evolution of $\left|V_{y}(x=0, y=0, z)\right| z$-profile in time. Thin line (spike in the middle) is at $t=0$, while thick and the thickest lines depict $\left|V_{y}(x=0, y=0, z)\right|$ at $t=5.0$ and 10.0. Here, $\alpha_{y}=1.0$.

case, as $\alpha_{y}=1$, it is 0.7188 ). Therefore, we can see that this way constructed analytic solution gives a tolerably good fit in Fig. 4. The deviation in the small $\eta$ end can be easily explained by the fact that the solution (A.10) was derived in the developed phase mixing approximation, which for such small values of $\eta$ would require numerical runs with end-simulation times much larger than $t=10$ (the latter is not possible with current computational facilities, as it would require further increase of the simulation box size). Thus, we conclude that the coupling to its compressive part does not alter the $1 / \sqrt{\eta}$ behavior of the Alfvénic part of a compressible MHD pulse.

In order to fully understand the dynamics of the Alfvénic part of the MHD pulse under our initial conditions as well as to appreciate fully the extent of the efficiency of the phase mixing for the coronal plasma heating, in Fig. 5 we show the evolution of the absolute value of the $z$-profile in the middle of the plasma inhomogeneity of $V_{y}$ with time. We gather from this graph that the initial, very localized, profile splits into two D'Alambert solutions propagating in opposite directions along the field (cf. Tsiklauri \& Nakariakov 2002), which then dissipate rapidly towards a diffuse, Gaussian shape.

Finally, we would like to reiterate the importance of plasma compressibility on the MHD wave dynamics in the context of wave-based theories of coronal heating and MHD turbulence (cf. Tsiklauri \& Nakariakov 2002). Our present numerical simulations confirm that when $\alpha_{y} \neq 0$, coupling to the Alfvénic part of the MHD pulse quite efficiently generates a density perturbation (which was initially zero) on a sizeable fraction of the initial Alfvén amplitude.

\section{Conclusions}

In summary, we have established that:

- Efficiency of the decay of the Alfvénic part of a compressible MHD pulse is related linearly to the degree of localization of the pulse in the homogeneous transverse direction, i.e. the coupling strength parameter $\alpha_{y}$.
- In the developed stage of phase mixing (for large times), coupling to its compressive part does not alter the $1 / \sqrt{\eta}$ behavior of the Alfvénic part of a compressible MHD pulse.

- The dynamics of Alfvén waves can still be qualitatively understood in terms of the previous 2.5D models, e.g., Hood et al. (2002a)'s law $\left(B_{y} \propto t^{-3 / 2}\right)$ for spatially localized, single pulses, survives in the case of 3D compressive MHD waves (in the absence of an ignorable coordinate).

- All of the above implies that phase mixing remains a relevant paradigm for the coronal heating applications in realistic 3D geometry and compressive plasma.

It is useful to give estimates of typical scales of the problem. We choose a typical (normalization) length scale of the transverse inhomogeneity $L=10 \mathrm{Mm}$ and Alfvén speed $C_{\mathrm{A}}=1000 \mathrm{~km} \mathrm{~s}^{-1}$, which are appropriate for example for coronal plumes. The coronal value for shear (Braginskii) viscosity, by which Alfvén waves damp, is estimated to be about $\eta=1 \mathrm{~m}^{2} \mathrm{~s}^{-1}$ (e.g. Hood et al. (2002a), which translates into $10^{-10}$ in the dimensionless units). However, as some recent studies suggest (Nakariakov et al. 1999; Ofman \& Aschwanden 2002) the actual value of the shear viscosity is possibly much larger due to micro-turbulence, e.g. $10^{9.2 \pm 3.5} \mathrm{~m}^{2} \mathrm{~s}^{-1}$ (Ofman \& Aschwanden 2002). Effectively, this implies that the Alfvén waves damp by bulk Braginskii viscosity rather than the shear one. The latter estimate is based on the observations of standing (kink) Alfvén waves in the coronal loops. Thus, its use in our estimates is justified provided the propagating Alfvén waves, studied here, are affected by the same dissipation mechanism as the standing ones. Using Eq. (A.9) (for $x=0$ ) we can estimate the characteristic time of damping of the amplitude of an Alfvén wave (when it reduces, say, $e$-times) due to phase mixing. Thus, we obtain approximately $2745.22 \mathrm{~s}$ for $\eta=1 \mathrm{~m}^{2} \mathrm{~s}^{-1}$ and $0.16-34.56 \mathrm{~s}$ for $\eta=10^{9.2 \pm 3.5} \mathrm{~m}^{2} \mathrm{~s}^{-1}$. This translates into $1647.13 \mathrm{Mm}$ (the Alfvén speed in the middle of inhomogeneity is $600 \mathrm{~km} \mathrm{~s}^{-1}$ ) for the damping length, for $\eta=1 \mathrm{~m}^{2} \mathrm{~s}^{-1}$, and $0.10-20.74 \mathrm{Mm}$ for $\eta=10^{9.2 \pm 3.5} \mathrm{~m}^{2} \mathrm{~s}^{-1}$. A similar analysis for the harmonic waves (now using Eq. (A.7) and substituting $k=2 \pi / \sigma)$ yields a damping length of $609.47 \mathrm{Mm}$ for $\eta=1 \mathrm{~m}^{2} \mathrm{~s}^{-1}$ and $0.04-7.67 \mathrm{Mm}$ for $\eta=10^{9.2 \pm 3.5} \mathrm{~m}^{2} \mathrm{~s}^{-1}$. These estimates would be affected if we choose different (probably higher) Alfvén speeds or if the pulse is wider. Also, note that in our numerical runs, the width of the Gaussian Alfvén pulse in the longitudinal $(z)$ direction was fixed at $\sigma \approx 0.03$, i.e. $0.3 \mathrm{Mm}$, while in the transverse $(y)$ direction the width, $1 / \sqrt{2} \alpha_{y}$, was varied from $+\infty$ down to 0.35 , i.e. from $+\infty$ down to $3.5 \mathrm{Mm}$.

Acknowledgements. DT acknowledges financial support from PPARC. Numerical calculations of this work were performed using the PPARC funded Compaq MHD Cluster at St Andrews. GR acknowledges financial support from a Leverhulme Emeritus Fellowship.

\section{Appendix A}

Here, we present an alternative, more rigorous, way to derive the power-law decay of $B_{y}$, suggested by (Hood et al. 2002a). If the initial Alfvénic perturbation (perturbing $V_{y}$ and $B_{y}$ ) does 
not depend on the $y$-coordinate, the linearized version of the system of Eqs. (1)-(3) allows for a complete decoupling of Alfvénic and compressive perturbations. Therefore, in order to investigate phase mixing quantitatively, we solve the following initial value problem with zero-gradient boundary conditions:

$\left[\partial_{t t}^{2}-C_{\mathrm{A}}(x)^{2} \partial_{z z}^{2}\right] B_{y}=\eta \partial_{t} \partial_{x x}^{2} B_{y}$,

$\left.B_{y}\right|_{t=0}=f(z)$.

Here, $C_{\mathrm{A}}(x)$ is a dimensionless Alfvén speed. In our numerical simulations we have used the following profile for $C_{\mathrm{A}}(x)$ : $C_{\mathrm{A}}(x)=1$ for $x<-0.5, C_{\mathrm{A}}(x)=0.6+0.4 \cos [\pi(x+0.5)]$ for $x \in[-0.5,0.5]$, and $C_{\mathrm{A}}(x)=0.2$ for $x>0.5$ (we employ here the same normalization as in Tsiklauri \& Nakariakov 2002 and, $\eta$ is normalized to the product of reference length and reference Alfvén speed as in Hood et al. 2002a). Note, that as in the developed stage of phase mixing (for large times) $\partial_{x x}^{2} \gg \partial_{y y}^{2}, \partial_{z z}^{2}$, in the forthcoming analytic treatment, we only retain largest (over $x$-coordinate) derivatives in the Laplacian (while for generality we still keep the full Laplacian in our numerical simulations, see below). Next, we introduce the following Lagrangian coordinates and slow (dissipation) time scale: $\bar{x}=x, \xi=z-C_{\mathrm{A}}(x) t, \tau=\varepsilon t$, with $\varepsilon \ll 1$. In these variables, the leading term on the left hand side of Eq. (A.1) is $-2 \varepsilon C_{\mathrm{A}}(x) \partial_{\tau \xi}^{2} B_{y}$, while on the right hand side the leading term is $-\eta C_{\mathrm{A}}(x) \partial_{\xi}\left[C_{\mathrm{A}}^{\prime}(x)^{2}(\tau / \varepsilon)^{2} \partial_{\xi \xi}^{2} B_{y}\right]$. Here, prime denotes a derivative over $x$. After integration over $\xi$ and introduction of yet another auxiliary variable, $s=\eta C_{\mathrm{A}}^{\prime}(x)^{2} \tau^{3} /\left(6 \varepsilon^{3}\right)=\eta C_{\mathrm{A}}^{\prime}(x)^{2} t^{3} / 6$, we obtain the following (diffusion) equation for $B_{y}$ :

$\partial_{s} B_{y}=\partial_{\xi \xi}^{2} B_{y}$,

which can be easily integrated:

$B_{y}=\frac{1}{2 \sqrt{\pi s}} \int_{-\infty}^{+\infty} \exp \left[-\frac{\left(\xi-\xi^{\prime}\right)^{2}}{4 s}\right] B_{y}\left(\xi^{\prime}, t=0\right) \mathrm{d} \xi^{\prime}$.

From Eq. (A.4) it immediately follows that in the developed stage of phase mixing, i.e. when $t, s \rightarrow+\infty$, the solution reduces to

$B_{y}=\frac{1}{2 \sqrt{\pi s}} \int_{-\infty}^{+\infty} B_{y}\left(\xi^{\prime}, t=0\right) \mathrm{d} \xi^{\prime}$.

Therefore, provided the integral

$\int_{-\infty}^{+\infty} B_{y}\left(\xi^{\prime}, t=0\right) \mathrm{d} \xi^{\prime}$

is finite (which is not always so e.g. for the harmonic initial conditions, see below), $B_{y}$ scales as $1 / \sqrt{s}$ or equivalently $B_{y} \propto$ $\eta^{-1 / 2} t^{-3 / 2}$

If we substitute a harmonic wave with initial conditions $B_{y}\left(\xi^{\prime}, t=0\right)=\exp \left(i k \xi^{\prime}\right)$ into Eq. (A.4), simple integration yields

$B_{y}=\mathrm{e}^{-\eta C_{\mathrm{A}}^{\prime}(x)^{2} t^{3} k^{2} / 6} \mathrm{e}^{-i k\left(z-C_{\mathrm{A}}(x) t\right)}$, thus, we recover the well-known Heyvaerts \& Priest's solution. For the spatially localized (in $z$ ) initial conditions

$\left.B_{y}\right|_{t=0}= \begin{cases}1+\cos (10 \pi z) & z \in[-0.1,0.1] \\ 0 & \text { elsewhere }\end{cases}$

following Hood et al. (2002a) we expand $B_{y}\left(\xi^{\prime}, t=\right.$ $0)$ in terms of Hermite polynomials $B_{y}\left(\xi^{\prime}, t=0\right)=$ $\sum_{n=0}^{\infty} \alpha_{n} \mathrm{e}^{-\xi^{\prime}} / 2 \sigma^{2} \mathcal{H}_{n}\left(\xi^{\prime} / \sigma\right)$ retaining only the fundamental mode $n=0$, i.e. to a fairly good accuracy initial conditions (Eq. (A.8)) can be approximated $B_{y}\left(\xi^{\prime}, t=0\right)=\alpha_{0} \mathrm{e}^{-\xi^{\prime 2} / 2 \sigma^{2}}$ (note that $\mathcal{H}_{0}=1$ ). Therefore, substituting the latter expression for $B_{y}\left(\xi^{\prime}, t=0\right)$ into the integral (A.4), and evaluating the integral analytically we obtain the solution of Eq. (A.1) to the leading (fundamental $n=0$ ) order

$B_{y}=\frac{\alpha_{0}}{\sqrt{1+\eta C_{\mathrm{A}}^{\prime}(x)^{2} t^{3} / 3 \sigma^{2}}} \exp \left[-\frac{\left(z-C_{\mathrm{A}}(x) t\right)^{2}}{2\left(\sigma^{2}+\eta C_{\mathrm{A}}^{\prime}(x)^{2} t^{3} / 3\right)}\right]$,

which coincides with the one obtained by Hood et al. (2002a). Here, $\sigma=0.033$ and $\alpha_{0}=1 / 5 \sqrt{2 \pi} \sigma$. For large time, $t$, Eq. (A.9) implies that the amplitude of the Alfvén wave (e.g. amplitude of the $B_{y}$ ) decays as

$B_{y}=\frac{1}{5}\left[2 \pi \eta C_{\mathrm{A}}^{\prime}(x)^{2} / 3\right]^{-1 / 2} t^{-3 / 2}$,

i.e., in the case of a localized in $z$-coordinate Alfvén pulse, Heyvaerts \& Priest's exponential decay is replaced by the power law $B_{y} \propto t^{-3 / 2}$ (Hood et al. 2002a).

Although, the both derivations in the end lead to the same answer, in our opinion, the one presented here is more rigorous, concise and it gives the general solution (Eq. (A.4)) and its asymptotic form (Eq. (A.5)) at once.

\section{References}

Arber, T. D., Longbottom, A. W., Gerrard, C. L., \& Milne, A. M. 2001, J. Comput. Phys., 171, 151

Botha, G. J. J., Arber, T. D., Nakariakov, V. M., \& Keenan, F. P. 2000, A\&A, 363, 1186

Heyvaerts, J., \& Priest, E. R. 1983, A\&A, 117, 220

Hood, A. W., Brooks, S. J., \& Wright, A. N. 2002a, Proc. Roy. Soc. Lond. A, 458, 2307

Hood, A. W., Brooks, S. J., \& Wright, A. N. 2002b, Proc. Roy. Soc. Lond. A, accepted

Malara, F., Primavera, L., \& Veltri, P. 1996, ApJ, 459, 347

Nakariakov, V. M., Ofman, L., DeLuca, E. E., Roberts, B., \& Davila, J. M. 1999, Science, 285, 862

Nakariakov, V. M., Roberts, B., \& Murawski, K. 1997, Sol. Phys., 175, 93

Nakariakov, V. M., Roberts, B., \& Murawski, K. 1998, A\&A, 332, 795

Ofman, L., \& Aschwanden, M. J. 2002, ApJ, 576, L153

Parker, E. N. 1991, ApJ, 376, 355

Roussev, I., Galsgaard, K., Erdelyi, R., \& Doyle, J. G. 2001, A\&A, 370,298

Tsiklauri, D., Arber, T. D., \& Nakariakov, V. M. 2001, A\&A, 379, 1098

Tsiklauri, D., Nakariakov, V. M., \& Arber, T. D. 2002, A\&A, 395, 285

Tsiklauri, D., \& Nakariakov, V. M. 2002, A\&A, 393, 321 